methods for diagnosis of Neisseria gonorrhoeae and Trichomonas vaginalis infections. However, PCR was available for testing for Chlamydia trachomatis, N. gonorrhoeae, Mycoplasma genitalium, Gardnerella vaginalis, $M$. hominis and Ureaplasma urealyticum using Russia-produced diagnostic tests. Serology remained in use for diagnosis of chlamydial infection and trichomoniasis. No appropriate and complete quality assurance and control system was available.

Conclusions In Tver, Russia, the detection of several STI agents has to be optimised, and international evidence-based standards and appropriate quality management systems introduced. Beneficially, the laboratory diagnosis is further centralised, which makes it easier to implement appropriate international evidence-based STI guidelines.

\section{P2.024 EVALUATION OF THE GENITAL MICROBIOTA IN MEN AND WOMEN USING AN AUTOMATED SYSTEM FOR ANALYSIS OF MICROSCOPY IMAGES OF WET AND STAINED SMEARS}

doi:10.1136/sextrans-2013-051184.0289

E Rybina, E Bulatova, Z Martikainen, S Zatsiorskaya, M Zaitseva, V Panteleyev. A Savicheva, Eastern European Network for SexualReproductive Health, Ott Institute of Obstetrics and Gynecology Academy of Medical Sciences of Russian Federation, St Petersburg, Russian Federation

Background In Russia, microscopy of the genital microbiota in both women and men are performed at laboratory, not at physician's office. Microscopy is a simple and cheap method, which, together with a clinical presentation, provides sufficient information for diagnosis of bacterial vaginosis, vulvovaginal candidiasis, trichomoniasis, cervicitis, urethritis. Improvement of microscopy diagnostic methods with the use of automated computerised system is important.

Methods Clinical samples were obtained from 100 men and 150 women of reproductive age. In total, 150 vaginal, cervical and female urethral samples, 73 male urethral samples and 17 prostatic samples were analysed using microscopy of Gram and methylene blue stained preparations. The presence of polymorphonuclear leukocytes, lactobacilli, "clue" cells, yeasts, trichomonads, grammenegative diplococci was assessed. Vaginal samples were also assessed for bacterial vaginosis using the Nugent score. For analysis, image analyzer including a Biological Microscope MT5000 Series, Neiji Techno Co., Ltd (Japan), digital colour camera Progress CT3 and software UroGyn were used.

Results In $17.33 \%, 11.33 \%$ and $2 \%$ of the vaginal samples, "clue" cells, yeasts and trichomonads were visualised by microscopy. Signs of cervicitis were detected in $2 \%$ of the women, in none of the female samples signs of urethritis were seen. In men, signs of urethritis were revealed in $3.45 \%$, and prostatitis - in $29.4 \%$ of the samples. In none of the samples, Gram-negative diplococci were detected. There was complete agreement between the results obtained using the image analyzer and those obtained using traditional light microscopy.

Conclusion An automated system for the analysis of images obtained by microscopy investigation of urogenital samples from women and men is easy to use, allows documentation of results and facilitates their interpretation.

\section{P2.025 ETIOLOGY OF RESPIRATORY TRACT INFECTION IN HIV/ AIDS PATIENTS AT THE NATIONAL HOSPITAL OF TROPICAL DISEASES (NHTD) HANOI, VIETNAM}

doi:10.1136/sextrans-2013-051184.0290

L T Nguyen, ${ }^{2} \mathrm{M}$ V Nguyen, ${ }^{2} \mathrm{H}$ T X Trinh. ' National Hospital of tropical diseases, Hanoi, Viet Nam; '2Army medical academy, Hanoi, Viet Nam

Background Respiratory tract infection is leading causes of death among patients infected with human immunodeficiency virus in Vietnam. Identification the agents caused RTIs is very important to give specific treatment and to reduce mortality rate among HIV/ AIDS patients suffering from RTI.
Methods We conducted a cross-sectional study of 170 HIV/AIDS patients with signs and symptoms (clinical manifestations and/or broncho aveolar lesions through X-ray chest film) of respiratory tract to indentify the common agents by analysing bronchoaveolarlavage (BAL).

Results A total of 170 HIV/AIDS patients (138 male and 32 female) involved in the study and 170 BAL samples had been taken to identify the common agents caused RTIs. 148/170 (87.1\%) patients had been diagnosed RTIs with following agents: Mycobacterium tuberculosis 79/148 (53.4\%), PJP 12/148 (8.1\%), bacteria 59/148 (39.9\%), fungi 54/148 (36.5\%) and CMV 2/148 (1.4\%). 52/148 (35.1\%) patients had been isolated 2 differential agents at a moment. Most patients have very low $\mathrm{CD} 4+$ count $\left(80.4 \% \leq 100 \mathrm{cells} / \mathrm{mm}^{3} ;\right.$ mean $=74.6$; $\mathrm{SD}=118.7$; median $=22$ ). The more common bacteria are: Pseudomonas (P.aeruginosa, P.putida, P.pneumotropica) 15/59 (25.4\%), Streptococcus (S.pneumoniae, S.pyogene) 11/59 (18.6\%), Acinobacter (Aci baumani, Aci.juni, Aci.minimus) 6/59 (10.2\%), E.coli 3/59 (5.1\%) and S.aureus $3 / 59$ (5.1\%). Other include: H.influenza $2 / 59$ and each following spp have 1: Achromobacter xylosoxidans, K.pneumoniae, Enterobacter clocae, Moraxella catarhalis, and Rhodococcus equi. Isolated fungal spp include: Candida albicans 32/54 (59.2\%), Penicillium marneffei 14/54 (25.9\%), Aspergilus spp 4 (7.4\%), Candida spp 3/54 (5.6\%) and Cryptococcus neoformans 1/54 (1.9\%). The common complex infections are MTB-Fungi (16 patients), MTB-Bacteria (14 patients) and Bacteria-Fungi (11patients).

Conclusion Mycobacterium tuberculosis, bacteria (P.aeruginosa, P.putida, P.pneumotropica, S.pneumoniae, S.pyogene, and Aci. baumani) and fungi (Candida albicans and Penicillium marneffei) are the more common in HIV/AIDS patients who have RTIs. Because of advanced immune depression, patients may have complex infections in a moment.

\section{P2.026 AUDIT OF ADHERENCE TO UK NATIONAL GUIDELINE FOR MANAGEMENT OF EPIDIDYMO-ORCHITIS}

doi:10.1136/sextrans-2013-051184.0291

C Whitfield, S Rodgers. Manchester Centre for Sexual Health, Manchester, UK

Background The UK guideline for epididymo-orchitis management makes recommendations about appropriate diagnostic tests, treatment regimes and health promotion principles. This audit compares our clinic's performance with reference to these guidelines.

Methods Case notes of patients diagnosed with epididymo-orchitis over seven months were reviewed. Details of sexual orientation, investigations, treatment and management were recorded.

Results There were 84 patients comprising 61(71.2\%) heterosexual men, $22(26.2 \%)$ men who had sex with men (MSM), and $1(1.2 \%)$ bisexual man. Fifty one (60.7\%) men had all four recommended microbiological investigations of gramme stained urethral smear, urethral swab for $N$. gonorrhoeae culture, first pass urine (FPU) for nucleic acid amplification tests for $N$. gonorrhoeae and C. trachomatis, and microscopy and culture of mid-stream urine (MSSU) for bacteria. Of the 84 patients, $81(96.4 \%)$ had 3 tests taken of urethral smear, swabs and FPU, but MSSU was only performed in $57(67.9 \%)$ men. Ten $(11.9 \%)$ men were diagnosed with chlamydia and $3(3.6 \%)$ with gonorrhoea, and $82(97.6 \%)$ patients received appropriate antibiotic treatment according to the guidelines. Advice on rest, analgesia and scrotal support was poorly documented $(9.5 \%$; $9.5 \%$; $8.3 \%$ respectively) Guidance about abstinence from sex and partner notification (PN) was documented in $65(77.4 \%)$ cases and explanation about the condition recorded in $54(64.3 \%)$ cases. A leaflet was offered in $3(3.6 \%)$ cases. Seventy six $(90.5 \%)$ patients had a follow up appointment offered at 2 weeks but only $42(55.3 \%)$ patients attended. At the review, PN was completed for all patients. A written action plan was recorded in $100 \%$ of men with ongoing symptoms. 